\title{
Calidad de vida relacionada con la salud en sujetos con VIH
}

\author{
Health-Related quality of life in people with HIV \\ Qualidade de vida relacionada à saúde em sujeitos com HIV
}

\author{
Carlos Jesús Canova Barrios $^{\text {a }}$ \\ Universidad de Ciencias Empresariales y Sociales (UCES), \\ Argentina \\ carlos.canova1993@gmail.com \\ ORCID: https://orcid.org/0000-0003-3901-6117
}

DOI: https://doi.org/10.11144/Javeriana.ie23.cvrs

Recibido: 21 Abril 2021

Aceptado: 17 Noviembre 2021

Publicado: 30 Diciembre 2021

\section{Resumen:}

Introducción: Desde la identificación del VIH se ha avanzado en el desarrollo de tratamientos más seguros y eficaces que aseguren la sobrevida de los sujetos afectados, siendo el reto de los profesionales sanitarios el mejoramiento de la calidad de vida relacionada con la salud de estos. Objetivo: Analizar la calidad de vida relacionada con la salud de una muestra de pacientes con VIH de Buenos Aires, Argentina. Método: Estudio analítico, transversal y cuantitativo realizado durante los meses de enero a marzo de 2020. Participaron un total de 144 sujetos. Se administraron los cuestionarios SF-36 y Morisky-Green. Resultados: La muestra estuvo comprendida mayoritariamente por hombres, solteros, sin hijos y con estudios universitarios. El 49,30 \% presentaba una buena adherencia al tratamiento. La Función física fue la dimensión mejor evaluada $(92,19)$, mientras que la Vitalidad fue la peor percibida $(61,42)$. El componente mental fue el más afectado. El sexo femenino, nivel de estudios primario, edad más alta, tenencia de hijos y la presencia de complicaciones se asociaron a una peor calidad de vida. Conclusiones: Se hace importante implementar acciones tendientes a mejorar la adherencia al tratamiento de los pacientes y promover estilos de vida favorecedores de la salud. Asimismo, el abordaje de los sujetos ha de ser integral, considerando las dimensiones psíquica, emocional, social y espiritual como parte de las áreas de atención. La calidad de vida debe ser un indicador de seguimiento para los profesionales sanitarios.

Palabras clave: calidad de vida, VIH, Síndrome de Inmunodeficiencia Adquirida, cumplimiento y adherencia al tratamiento.

\section{Abstract:}

Introduction: Since the HIV was identified, more efficacious and safer treatments have been developed in order to ensure survival of people with the virus. The challenge to the health professionals is to improve the quality of life in relation to these patients' health condition. Objective: To analyze the health-related quality of life in a sample of HIV patients from Buenos Aires (Argentina). Methods: It is a quantitative, cross.-sectional, analytical study conducted from January to March 2020. In total, 144 subjects took part in this study. Questionnaires SF-36 and Morisky-Green were applied. Results: Most of the sample consisted of male subjects, single, without children and with college education. The physical function evaluation reported the best values (92.19), while vitality showed the worse evaluation (61.42). The mental component was the most affected one. Female gender, low schooling, been older, having children and health complications were associated with a worse quality of life. Conclusions: It is quite important to implement actions intended to improve the treatment adherence among the patients and to encourage styles of life that favor their health. Likewise, health professional must approach these patients in a comprehensive way, considering the psychic, emotional, social and spiritual dimensions as a part of the healthcare. The quality of life must be taken as a follow-up indicator by the health professionals.

Keywords: quality of life, HIV, Immunodeficiency acquired syndrome, treatment compliance and adherence.

\section{Resumo:}

Introdução: Desde a identificação do VIH houve avanços no desenvolvimento de tratamentos mais seguros e eficazes que garantam a sobrevivência dos sujeitos acometidos, sendo o desafio para os profissionais sanitários o melhoramento da qualidade de vida relacionada à saúde destes. Objetivo: Analisar a qualidade de vida relacionada à saúde de uma amostra de pacientes com HIV de Buenos Aires, Argentina. Método: Estudo analítico, transversal e quantitativo realizado durante os meses de janeiro a março de 2020. Participara um total de 144 sujeitos. Foram aplicados os questionários SF-36 e Morisky-Green. Resultados: a amostra foi composta principalmente por homens, solteiros, sem filhos e com estudos universitários. $49,30 \%$ tiveram boa adesão ao tratamento. A Função física foi a dimensão melhor avaliada $(92,19)$, enquanto Vitalidade foi a pior percebida $(61,42)$. O componente mental foi o mais afeitado. O sexo feminino, nível de escolaridade fundamental, idade mais avançada, tenência de filhos e presença de

Notas de autor 
complicações foram associadas à pior qualidade de vida. Conclusões: É importante implementar ações que visem melhorar a adesão ao tratamento dos pacientes e promover estilos de vida saudáveis. Da mesma forma, a abordagem dos sujeitos deve ser integral, considerando as dimensões psíquica, emocional, social e espiritual como parte das áreas de atenção. A qualidade de vida deve ser um indicador de monitoramento para os profissionais sanitários.

Palavras-chave: Qualidade de vida, HIV, Síndrome de Imunodeficiência Adquirida, cumprimento e adesão ao tratamento.

\section{Introducción}

La Organización Mundial de la Salud (1) define lasalud como "un estado de completo bienestar físico, emocional y social, y no solamente la ausencia de afecciones o enfermedades", y la calidad de vida como "la percepción del individuo de su posición en la vida en el contexto de la cultura y sistema de valores en los que vive y en relación con sus objetivos, expectativas, estándares y preocupaciones" (2), siendo integrada por elementos como la salud física, el estado psicológico, el nivel de independencia, las relaciones sociales y la relación con el ambiente que lo rodea. La integración de estos dos términos define la calidad de vida relacionada con la salud (CVRS), que refiere al impacto que tiene una enfermedad y su tratamiento sobre la percepción de la satisfacción con el bienestar físico, psíquico, social y espiritual.

La CVRS es definida como el nivel de bienestar derivado de la evaluación que la persona realiza de diversos dominios de su vida, considerando el impacto que en estos tiene su estado de salud, es decir, es una medida de percepción que manifiesta el paciente sobre elementos psíquicos y físicos que son relevantes para su bienestar. Por su parte, la definición dada por (3) contempla la percepción subjetiva, influenciada por el estado de salud actual y de la capacidad para realizar aquellas actividades importantes para el individuo.

Podrían referirse dentro de los propósitos de las mediciones de la CVRS: el monitoreo de la salud de las personas, la evaluación de las políticas de asistencia social y sanitaria, la realización del diagnóstico de la naturaleza, severidad y pronóstico de una enfermedad, la focalización los recursos en función de las necesidades detectadas (incluyendo el tratamiento), y en conjunto, la evaluación de los efectos de un tratamiento (4).

En términos generales, la CVRS integra tanto aspectos objetivos de la salud (funcionalidad física, interacción con el medio) como aspectos subjetivos (incluyendo la satisfacción general y la percepción de la propia salud).

Desde su descubrimiento, la infección por el virus de inmunodeficiencia humana (VIH) ha avanzado hasta convertirse en una enfermedad crónica, si bien con el pasar del tiempo y los avances en el desarrollo de fármacos se ha mejorado considerablemente la sobrevida de los afectados, con lo cual se ha convertido a la CVRS en un área de interés en el marco de la atención en salud de esta población (5). De acuerdo con la Organización Mundial de la Salud, para finales de 2019 la infección por VIH y el SIDA (Síndrome de Inmunodeficiencia Adquirida) había cobrado casi 33 millones de vidas, y se estimó que a nivel mundial 38 millones de personas vivían con este virus, de las cuales 136000 casos se registraban en la República Argentina, con una prevalencia estimada del $0,4 \%$ entre jóvenes y adultos de toda la población (6).

Estudios realizados en población con VIH han mostrado que los niveles de CVRS son afectados por factores como depresión y ansiedad, preocupación financiera y falta de soporte económico, falta de soporte social (amigos y familia), sintomatología asociada a la enfermedad, consumo de drogas y alcohol, estigmatización, mala relación con los profesionales tratantes y una menor accesibilidad al sistema de salud, la medicación y otras prestaciones sanitarias (7-9). Sin embargo, otras características como el sexo femenino (10), bajo nivel educativo (11) y homosexualidad (12) son aspectos relacionados con una peor evaluación de la CVRS.

En cuanto al conteo de células CD4 y la carga viral, se han descrito algunas tendencias claras que apuntan a una mejor evaluación de la CVRS en los pacientes con mayor conteo de células (9,13-16), y una peor evaluación en aquellos con signos de inmunosupresión (17). Sin embargo, algunos estudios no han 
identificado asociación alguna entre la CVRS y el conteo de CD4 (18). Tampoco se ha identificado la carga viral indetectable como predictor de mejor CVRS (7); en contraste, algunos trabajos refieren que algunas áreas como la función social, la depresión, la ansiedad y la satisfacción con la calidad de vida sí correlacionan negativamente con la carga viral elevada y las complicaciones asociadas $(9,19,20)$.

Respecto al uso de la terapia antirretroviral de gran actividad (TARGA), se ha identificado un impacto positivo de esta sobre la CVRS, sobre todo cuando los pacientes reciben el primer régimen terapéutico, algo posiblemente relacionado con la recomposición del sistema inmune y la mejoría de diversidad de síntomas asociados a la infección $(21,22)$. Sin embargo, se ha asociado el deterioro de la CVRS con la presencia de efectos adversos relacionados con la medicación, como la lipodistrofia $(9,23)$.

Por lo anterior, es de esperarse que, en los pacientes con mejor nivel de adherencia a la TARGA, se hallen mayores niveles de CVRS, dado que de este modo obtendrán mejores beneficios de la terapéutica (mayores niveles de CD4, menor cantidad y severidad de síntomas, niveles bajos o indetectables de carga viral). Algunos estudios han identificado la relación mencionada (15), mientras que otros trabajos no han encontrado relación alguna (24).

Es por lo anterior que se diseñó el presente trabajo, con el objetivo de analizar la CVRS y sus factores relacionados en una muestra de sujetos que conviven con VIH en la Ciudad Autónoma de Buenos Aires, Argentina, en el periodo enero-marzo del 2020.

\section{Método}

Se desarrolló un estudio transversal, de tipo analítico-correlacional y con enfoque cuantitativo. El proceso de recogida de datos se realizó entre enero y marzo del 2020.

La muestra estuvo integrada por sujetos VIH positivos, atendidos en una institución privada de la Ciudad Autónoma de Buenos Aires, de ambos sexos, con edades entre los 18 y 60 años, sin alteraciones en el estado mental, con más de 6 meses de diagnóstico y con más de 3 meses de instauración de la TARGA. Se excluyó a gestantes, aquellos con terapia hormonal restitutiva activa, sujetos que hubiesen sido internados en los últimos 30 días previa recopilación de la información y aquellos que no desearan participar voluntariamente en el estudio. Se implementó el muestreo probabilístico aleatorio, usando la base de datos de consultantes. La muestra fue calculada teniendo en cuenta un nivel de confianza del $95 \%$, poder estadístico del $80 \%$, y proporción esperada del $20 \%$.

Para la medición de la CVRS se utilizó el cuestionario SF-36, un instrumento genérico, breve y de carácter autoadministrado, el cual cuenta con evidencia científica que sustenta su uso por encima de otros cuestionarios para medir la CVRS en poblaciones con enfermedades crónicas, incluida la infección por VIH (8), y con un alfa de Cronbach en sus escalas, que oscila entre 0,79 y 0,92 (25), siendo validado exitosamente para su uso en la población argentina.

El SF-36 consta de 36 reactivos respondidos en una escala tipo Likert que va desde 2 a 6 opciones de respuesta, y que se agrupan en 8 dimensiones: función física (10 reactivos), rol físico (4 reactivos), dolor corporal (2), salud general (5), vitalidad (4), función social (2), rol emocional (3) y salud mental ( 5 reactivos), con dos componentes (físico y mental). Uno de los ítems del instrumento no se incorpora a ningún componente ni dimensión, y se analiza bajo la denominación de "Transición del estado de salud”. Los puntajes resultantes del proceso de homogenización, cálculo de puntaje bruto, sumatoria y transformación lineal necesaria para el análisis del instrumento, arrojan índices que van entre 0 y 100 . Para su interpretación, puntuaciones superiores o inferiores a 50 indican mejor o peor estado de salud, respectivamente $(25,26)$.

Además, se implementó el cuestionario Morisky-Green-Levine para evaluar la adherencia al tratamiento farmacológico, el cual ha sido utilizado en estudios similares $(27,28)$, con un alfa de Cronbach de entre 0,6 y 
0,86. Este se integra por cuatro preguntas con opciones de respuesta dicotómica ( $\mathrm{Si} / \mathrm{No}$ ) y que caracterizan la adherencia como buena y mala.

Los instrumentos referidos previamente fueron complementados con una batería de preguntas cuyo objetivo fue describir sociodemográficamente a la muestra (sexo, edad, nivel educativo, estado laboral y tenencia de hijos), identificar el control inmunológico de la enfermedad (carga viral, CD4 y complicaciones) y algunas variables identificadas previamente como intervinientes (hábito tabáquico, realización de actividad física y adherencia al tratamiento).

Los instrumentos fueron entregados a los pacientes durante la consulta médica regular, posterior a la solicitud del consentimiento informado, y se generó un espacio para la resolución de dudas respecto a su participación. Este procedimiento se llevó a cabo en conjunto con el médico tratante y el investigador principal del estudio.

La información recolectada fue tabulada en una base de datos en el programa Microsoft Excel, y analizada posteriormente con el programa Infostat v/L. Dado que el SF-36 muestra sus resultados en forma de índice y que la distribución no respondía a los criterios de normalidad, se utilizaron pruebas no paramétricas para el análisis inferencial de los datos. La identificación de relaciones, correlaciones y asociaciones se realizó de acuerdo con los valores que asumieron las variables y los cruces que se estimó pertinentes realizar. Así, las pruebas U-Mann Whitney \& Kruskal Wallis se realizaron para el cruce de variables categóricas (dicotómicas y politómicas, respectivamente) con numéricas, como por ejemplo, al contrastar sexo, tenencia de hijos, hábito tabáquico o realización de actividad física con los puntajes de las dimensiones de la CVRS o los niveles de CD4. La prueba de Chi cuadrado $\left(\chi^{2}\right)$ se usó para el análisis de asociación entre variables categóricas versus categóricas, como por ejemplo, hábito tabáquico y sexo versus adherencia al tratamiento. Las correlaciones se establecieron entre variables numéricas versus numéricas, y se implementó el test de correlación de Spearman (R.) para identificar correlaciones entre variables numéricas como el conteo de CD4, carga viral y tiempo de evolución de la enfermedad frente a CVRS.

Respecto a las consideraciones éticas, se tuvieron en cuenta los criterios éticos presentes en la declaración de Helsinki. Se solicitó el consentimiento informado a los participantes, y se aseguró la confidencialidad de los datos personales en cumplimiento de la regulación local; se obtuvo la aprobación del estudio por parte del Comité de Ética para la Investigación Científica y Tecnológica.

\section{Resultados}

En este estudio participaron un total de 153 sujetos, pero se eliminaron 9 registros debido a que estaban incompletos, quedando una muestra de 144 .

La media de edad de los encuestados fue de 43,8 años (DT: 11), con una edad mínima de 24 y una máxima de 64 años. Los encuestados en su mayoría fueron hombres (82,6\%), solteros $(61,8 \%)$, con estudios universitarios $(50,0 \%)$, con empleo registrado en relación de dependencia $(58,3 \%)$ o autónomos $(26,3 \%)$ (véase tabla 1 ).

El tiempo de evolución de la enfermedad fue de 9,9 años (DT: 8, rango 1-31 años) y el 31,9 \% presentó alguna complicación, siendo la más usual la neumonía por distintos gérmenes en el 17,36 \% de la muestra. El 15,2 \% de los encuestados presentaron carga viral detectable, con una media de 3952,7 copias/mL (DT: 4147) y una media de CD4 de 655 células/mm. (DT: 287). 
TABLA 1

Variables sociodemográficas relacionadas con la enfermedad

\begin{tabular}{|c|c|c|}
\hline Variable & FA $(n)^{a}$ & FR $(\%)^{b}$ \\
\hline \multicolumn{3}{|c|}{ Sexo } \\
\hline Hombre & 119 & 82,6 \\
\hline Mujer & 25 & 17,3 \\
\hline \multicolumn{3}{|c|}{ Estado civil } \\
\hline Soltero & 89 & 61,8 \\
\hline Unión libre & 29 & 20,1 \\
\hline Casado & 19 & 13,1 \\
\hline Divorciado & 6 & 4,1 \\
\hline Viudo & $1 \mid$ & 0,6 \\
\hline \multicolumn{3}{|c|}{ Nivel educativo } \\
\hline $\begin{array}{l}\text { Sin educación - } \\
\text { primario }\end{array}$ & 5 & 3,47 \\
\hline Secundario & 32 & 22,2 \\
\hline Terciario & 35 & 24,3 \\
\hline Universitario & 72 & 50 \\
\hline \multicolumn{3}{|c|}{ Estado laboral } \\
\hline Empleado & 127 & 88,1 \\
\hline Desempleado & $17 \mid$ & 11,8 \\
\hline \multicolumn{3}{|c|}{ Tenencia de hijos } \\
\hline Sí & 37 & 25,6 \\
\hline No & 107 & 74,3 \\
\hline \multicolumn{3}{|c|}{ Complicaciones } \\
\hline Ninguna & 98 & 68 \\
\hline Neumonía & 25 & 17,3 \\
\hline Infección por herpes & 12 & 8,3 \\
\hline Candidiasis & 7 & 4,8 \\
\hline Sarcoma de Kaposi & 5 & 3,4 \\
\hline Tuberculosis & 4 & 2,7 \\
\hline Otra & 1 & 0,6 \\
\hline \multicolumn{3}{|c|}{ Hábito tabáquico } \\
\hline Sí & 56 & 38,8 \\
\hline No & 88 & 61,1 \\
\hline \multicolumn{3}{|c|}{ Actividad física } \\
\hline Sí, todos los días & 19 & 13,1 \\
\hline $\begin{array}{l}\mathrm{Si} \text {, tres veces por } \\
\text { semana }\end{array}$ & 62 & 43 \\
\hline No, nunca & \begin{tabular}{l|l}
63 \\
\end{tabular} & 43,7 \\
\hline \multicolumn{3}{|c|}{ Adherencia al tratamiento } \\
\hline Buena & 71 & 49,3 \\
\hline Mala & 73 & 50,6 \\
\hline
\end{tabular}

La prevalencia de tabaquismo fue del 38,8 \% y el sedentarismo ascendió al 43,7 \%. Al analizar la adherencia al tratamiento, se halló que el 43,0 \% olvida tomar su tratamiento, el 6,9\% no toma la medicación en la hora indicada, el 7,6 \% deja de tomar la medicación cuando se siente bien y el 10,4\% deja de tomar la medicación si esta le sienta mal. A partir de los datos anteriores, la adherencia al tratamiento fue caracterizada como mala en el 50,6\% de los sujetos.

$\mathrm{Al}$ analizar el comportamiento de la CVRS se encontró que la dimensión mejor percibida fue la Función física, con una media de 92,1 [DT: 13,9; IC95\%: 89,8-94,4], mientras que la dimensión con peor percepción fue la de Vitalidad, con una media de 61,42 [DT: 14,93; IC95 \%: 58,9-63,8]. El componente mental fue el más comprometido al presentar medias más bajas (tabla 2).

TABLA 2

Comportamiento de las dimensiones de la CVRS

\begin{tabular}{|c|c|c|c|c|}
\hline Componente & Dimensión & media $(D T)^{a}$ & IC95 $\%^{b}$ & Min-Max ${ }^{c}$ \\
\hline \multirow[b]{4}{*}{ Salud fisica } & Función fisica & $92,19(13,99)$ & $89,88-94,49$ & $25-100$ \\
\hline & \begin{tabular}{|l|} 
Rol fisico \\
\end{tabular} & $73,44(36,83)$ & $67,37-79,50$ & $0-100$ \\
\hline & Dolor corporal & $71,62(26,78)$ & $67,20-76,03$ & $12-100$ \\
\hline & Salud general & $74,19(18,47)$ & $71,14-77,23$ & $5-100$ \\
\hline \multirow[b]{4}{*}{ Salud mental } & \begin{tabular}{|l|} 
Vitalidad \\
\end{tabular} & $61,42(14,93)$ & $58,96-63,87$ & $30-90$ \\
\hline & Función social & $76,56(25,51)$ & $72,35-80,76$ & $0-100$ \\
\hline & Rol emocional & $68,06(43,16)$ & $60,95-75,16$ & $0-100$ \\
\hline & Salud mental & $61,64(18,70)$ & $58,54-64,73$ & $16-88$ \\
\hline $\begin{array}{c}\text { Transición de la } \\
\text { salud }\end{array}$ & $\begin{array}{l}\text { Evolución declarada } \\
\text { de la salud }\end{array}$ & $75.15(20.10)$ & $71.82-78.45$ & $40-100$ \\
\hline
\end{tabular}

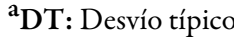

b IC95 \%: Índice de confianza al $95 \%$

${ }^{\mathrm{c}}$ Min-Max: Mínimo-Máximo.

Fuente: Elaboración propia 
Se caracterizaron los puntajes de las dimensiones de la CVRS en dos grupos, baja y alta, para puntajes entre 0-50 y 51-100, respectivamente. A partir de ello, se encontró que las dimensiones de Rol emocional y Rol físico presentaron prevalencias de baja CVRS en el 40,9\% y 34,7 \% de la muestra, respectivamente, mientras que en las dimensiones de Función física y Evolución declarada de la salud, solo en el 9,0 \% y 11,1 \% hubo una mala percepción, respectivamente (figura 1).

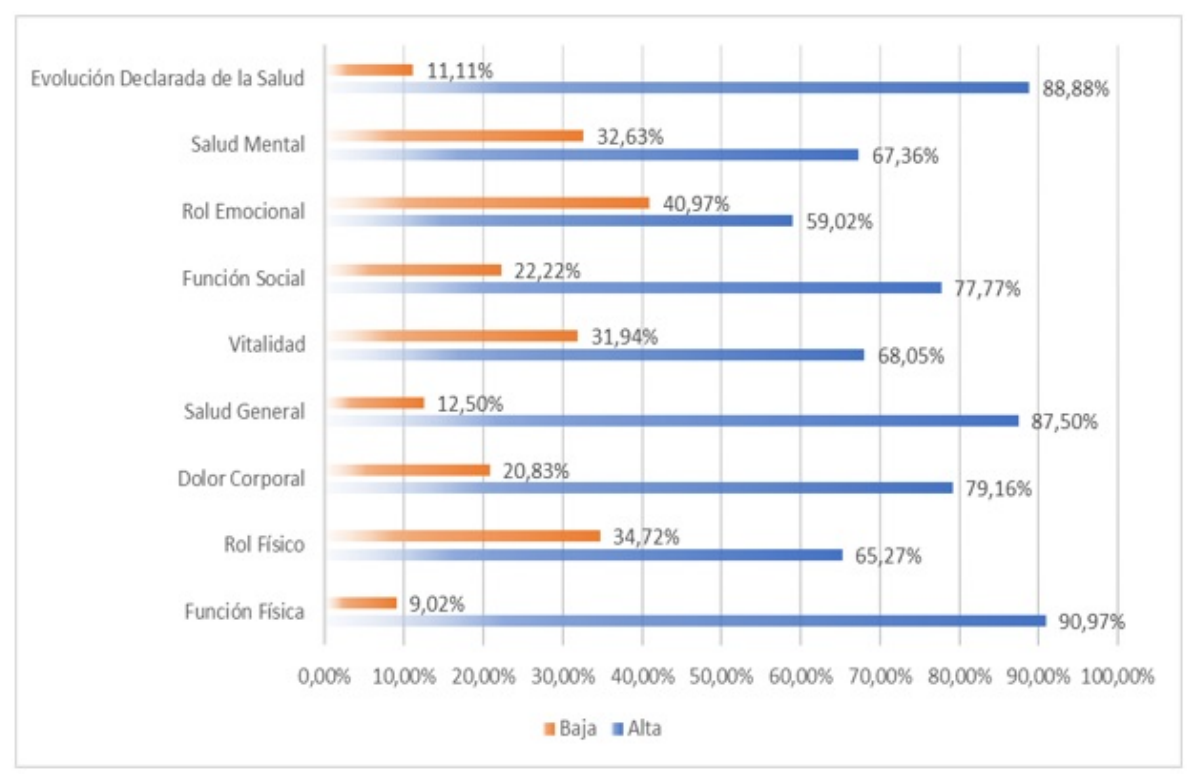

FIGURA 1

Evaluación de la CVRS

Fuente: Elaboración propia.

$\mathrm{Al}$ aplicar el test de correlación de Spearman, se identificaron correlaciones entre bajas y moderadas entre las variables de edad, tiempo de evolución de la enfermedad y carga viral con algunas de las dimensiones que componen la CVRS (tabla 3); no hubo correlación entre la CVRS y el conteo de CD4.

TABLA 3

Correlación entre edad, tiempo de evolución de la enfermedad, carga viral y CVRS

\begin{tabular}{|c|c|c|c|}
\hline Variables & Edad & $\begin{array}{l}\text { Tiempo de } \\
\text { evolución }\end{array}$ & Carga viral \\
\hline Función física & $\begin{array}{l}\text { rs: }-0,29, \\
p:<0,01\end{array}$ & $\begin{array}{l}\text { rs: }-0,33, \\
p:<0,01\end{array}$ & NS \\
\hline Rol físico & rs: $0,16, p: 0,04$ & rs: $-0,16, \mathrm{p}: 0,05$ & NS \\
\hline Dolor corporal & $\begin{array}{l}\text { rs: }-0,22, \\
p:<0,01\end{array}$ & $\begin{array}{l}\mathrm{rs}:-0,42, \\
\mathrm{p}:<0,01\end{array}$ & rs: $-0,19, \mathrm{p}: 0,02$ \\
\hline Salud general & $\mathrm{rs}: 0,25, \mathrm{p}:<0,01$ & NS & $\begin{array}{l}\text { rs: } 0,34, \\
\mathrm{p}:<0,01\end{array}$ \\
\hline Rol emocional & NS & NS & $\begin{array}{l}\text { rs: }-0,26, \\
\text { p: }<0,01\end{array}$ \\
\hline Salud mental & rs: $0,29, p:<0,01$ & NS & NS \\
\hline $\begin{array}{l}\text { Evolución } \\
\text { declarada de la } \\
\text { salud }\end{array}$ & NS & NS & rs: $-0,18, \mathrm{p}: 0,03$ \\
\hline Vitalidad & NS & NS & NS \\
\hline Función social & NS & NS & NS \\
\hline
\end{tabular}

NS: No significativo / No correlación.

Rs: Correlación.

Fuente: Elaboración propia

Los hombres presentaron medias superiores en las dimensiones de Función física (94,2 vs 82,2; p:0,02), Rol físico (77,7 vs 53,0; p:0,04) y Salud general (76,5 vs 62,9; p:<0,01). Respecto al estado civil, se encontró que los solteros presentaron medias superiores en la dimensión de Función física ( $\mathrm{p}:<0,01$ ); los casados en Rol 
físico ( $\mathrm{p}:<0,01)$, Vitalidad ( $\mathrm{p}: 0,01)$ y Rol emocional ( $\mathrm{p}:<0,01)$; y los divorciados en Salud general ( $\mathrm{p}:<0,01)$, Función social $(\mathrm{p}:<0,01)$ y Salud mental ( $\mathrm{p}: 0,01)$.

En el nivel de estudios, los universitarios presentaron medias superiores en las dimensiones de Función física (p:0,01), Rol físico (p:0,02), Salud general (p:0,03) y Rol emocional ( $\mathrm{p}:<0,01)$, y en aquellos con estudios terciarios, las medias superiores estuvieron en las dimensiones de Vitalidad (p:0,02), Salud mental ( $\mathrm{p}: 0,01)$ y Evolución declarada de la salud (p:0,04). Frente a la tenencia de hijos, con excepción de Salud mental y Evolución de la salud, las medias fueron inferiores en aquellos con hijos.

Respecto a la caracterización laboral, aquellos que tenían trabajo presentaron frente a los desempleados medias superiores y estadísticamente significativas en las dimensiones de Rol físico $(78,1$ vs 38,2; p: $<0,01)$, Salud general (75,8 vs 62,1; p:0,01), Rol emocional (71,3 vs 43,1; p:0,01), y Salud mental (62,9 vs 51,7; $\mathrm{p}: 0,04)$.

La presencia de complicaciones se relacionó con puntajes más altos en la CVRS en las dimensiones de Salud general ( $\mathrm{p}: 0,02)$, Rol emocional ( $\mathrm{p}: 0,02)$ y Evolución de la salud $(\mathrm{p}:<0,01)$, mientras que la Función física fue mayor en aquellos sin complicaciones $(\mathrm{p}:<0,01)$.

Se encontró que, en los no fumadores, las dimensiones de la CVRS presentaron puntajes más bajos $(\mathrm{p}:<0,01)$, exceptuando en Función física y Evolución de la salud. Aquellos con buena adherencia al tratamiento presentaron puntajes más altos en todas las dimensiones de la CVRS, con excepción de Dolor corporal y Rol físico.

Se identificó que los fumadores presentaron una buena adherencia en el 32,2 \% de las observaciones, mientras que, en los no fumadores, la adherencia ascendía al 60,2 \%, existiendo una relación entre ambas variables $\left(\chi^{2}: 9,70, \mathrm{p}:<0,01\right)$. El sexo y la adherencia al tratamiento no mostraron relación $\left(\chi^{2}: 0,13, \mathrm{p}: 0,71\right)$. La carga viral indetectable se relacionó con una mayor calidad de vida en todas las dimensiones, aunque estas diferencias fueron significativas solo en las dimensiones de Salud general ( $\mathrm{p}:<0,01)$, Rol emocional ( $\mathrm{p}:<0,01)$ y Evolución de la salud ( $\mathrm{p}: 0,05)$.

\section{Discusión}

La infección por VIH continúa siendo un importante problema de salud pública, dada la acelerada transmisión y el impacto para el sistema sanitario que representa el aumento en su prevalencia. Si bien en un inicio dicha infección era percibida inevitablemente como una enfermedad letal, con una supervivencia a los 3 años de entre el $5 \%$ y el $10 \%$, sumado al estigma social que representaba en su momento, hoy en día, el desarrollo de nuevas terapias más seguras y el mayor acceso a estas ha aumentado en gran medida la expectativa de vida, con lo que el SIDA pasó a ser una más de las enfermedades crónicas. Sumado a lo anterior, preocupa la prevalencia de factores de riesgo para el desarrollo de enfermedades crónicas no transmisibles a nivel nacional, más específicamente en la población con VIH. En el presente trabajo se encontró que el 38,8 $\%$ de los encuestados fumaban, lo cual supera la prevalencia de tabaquismo a nivel nacional, estimado en 22,2 \% (29), y predispone a esta población a diversas enfermedades que podrían afectar negativamente su CVRS.

Se halló que la carga viral detectable, la mala adherencia al tratamiento, el tabaquismo y el tiempo de evolución de la enfermedad se relacionaron con bajos niveles de CVRS, lo cual es corroborado por la literatura consultada (7,13-17); sin embargo, no se halló asociación entre los niveles de CD4 y la CVRS, similarmente a lo encontrado en otros estudios (18).

Los niveles de CVRS encontrados en las distintas dimensiones muestran en general un buen nivel percibido, similar a otros estudios (7). No se asoció la adherencia al tratamiento con los niveles de CD4/ $\mathrm{mm}^{3}$, aunque es sabido que es necesaria una buena adherencia para minimizar el riesgo de complicaciones o presencia de infecciones oportunistas $(9,19,20)$. Se resalta una adherencia detectada del $49,30 \%$, lo cual es un dato preocupante, similar a otros estudios con cifras de cumplimiento cercanas al $50 \%$ (30). 
El impacto de este punto sobre el tratamiento sobre la CVRS pudo ser evaluado, identificando que la buena adherencia incide en una mejor percepción de los aspectos de Salud general, Vitalidad, Función social, Rol emocional, Evolución de la salud y Salud mental. En ello se coincide con la literatura consultada, en la cual se relaciona la adherencia al tratamiento con la supresión viral, menor riesgo de complicaciones y mejor respuesta inmune (7,13-20).

Dados los hallazgos, se hace necesario desde la política sanitaria implementar acciones tendientes a mejorar la adherencia al tratamiento de los pacientes y promover estilos de vida favorecedores de la salud, incluyendo la realización de actividad física regular, la cesación del hábito tabáquico, la prevención del alcoholismo y las conductas de autocuidado de la salud. Los sistemas sanitarios han de tener una actitud proactiva hacia la medición de la CVRS, interviniendo los elementos que la afecten y propendiendo por una atención integral que contemple la multidimensionalidad del ser humano.

\section{Conclusión}

$\mathrm{Al}$ indagar sobre el comportamiento de las dimensiones de la CVRS se halló que la dimensión de Función física fue la mejor percibida (Media: 92,1; DT: 13,9), mientras que la dimensión Vitalidad fue la peor (Media: 61,42; DT: 14,93). La edad, el tiempo de evolución de la enfermedad y la carga viral mostraron correlaciones con la CVRS.

Algunas características que se relacionaron con mejores niveles de CVRS fueron el sexo masculino, el estado civil soltero, el nivel de educación superior, el no tener hijos, el ser laboralmente activo (empleado), el no fumar y el tener una buena adherencia al tratamiento; el tabaquismo se relacionó con una baja adherencia al tratamiento. Las dimensiones que integran el componente mental presentaron medias inferiores al ser comparadas con las del componente físico.

Finalmente, se reitera que es fundamental el mantenimiento de las intervenciones de promoción de la salud y prevención de la enfermedad dentro de todos los niveles de atención destinados a la población VIH-positiva, haciendo hincapié en la implementación de servicios especializados del primer nivel de atención, para que se promueva la adherencia al tratamiento y se mejore la calidad de vida percibida.

\section{Referencias}

1. Organización Mundial de la Salud. Constitución de la OMS [Internet]. 2014. https://apps.who.int/gb/bd/PDF/ bd48/basic-documents-48th-edition-sp.pdf\#page=7.

2. Organización Mundial de la Salud. ¿Qué calidad de vida? [Internet]. 1996. https://apps.who.int/iris/bitstream/handle/10665/55264/whf_1996_17_n4_p38;jsessionid=AD1C4 EA5EBBA1032E5A14F26AE5415D5?sequence $=1$

3. Shumaker SA, Ellis S, Naughton MJ. Assessing health-related quality of life in HIV disease: key measurement issues. Quality of Life Research. 1997;6:475-80. https://doi.org/10.1023/A:1018499813912.

4. Mokhatri-Hesari P, Montazeri A. Health-related quality of life in breast cancer patients: review of reviews from 2008 to 2018. Health Qual Life Outcomes. 2020:18,338. https://doi.org/10.1186/s12955-020-01591-x.

5. Reinius M, Wiklander M, Wettergren L, Svedhem V, Eriksson LE. The Relationship Between Stigma and HealthRelated Quality of Life in People Living with HIV Who Have Full Access to Antiretroviral Treatment: An Assessment of Earnshaw and Chaudoir's HIV Stigma Framework Using Empirical Data. AIDS Behav. 2018;22:3795-3806. https://doi.org/10.1007/s10461-018-2041-5.

6. Ministerio de Salud de la Nación, Argentina. Boletín Oficial Sobre VIH, SIDA y ITS N³7, año XXIII, diciembre 2020 [Internet]. Disponible en: https://bancos.salud.gob.ar/recurso/boletin-sobre-el-vih-sida-e-its-en-la-argen tina-ndeg-37. 
7. Cabrera S, Arteta Z, Meré J, Cavalleri F. Calidad de vida relacionada a la salud en personas con VIH y factores asociados. Rev Méd Urug. 2018;34(1):7-19. https://doi.org/10.29193/rmu.34.1.1

8. Cooper V, Clatworthy J, Harding R, Whetham J; Emerge Consortium. Measuring quality of life among people living with HIV: a systematic review of reviews. Health Qual Life Outcomes. 2017;15(1):220. https://doi.org/10.11 86/s12955-017-0778-6.

9. Ghiasvand H, Waye KM, Noroozi M, Ghaedamini Harouni G, Armoon B, Bayani A. Clinical determinants associated with quality of life for people who live with HIV/AIDS: a Meta-analysis. BMC Health Serv Res. 2019;19:768. https://doi.org/10.1186/s12913-019-4659-z

10. Lifson AR, Grandits GA, Gardner EM, Wolff MJ, Pulik P, Williams I, et al. Quality of life assessment among HIV-positive persons entering the INSIGHT Strategic Timing of AntiRetroviral Treatment (START) trial. HIV Med. 2015;16(Suppl. 1):88-96. https://doi.org/10.1111/hiv.12237.

11. Ballester-Arnal R, Gómez-Martínez S, Fumaz CR, González-García M, Remor E, Fuster MJ. A spanish study on psychological predictors of quality of life in people with HIV. AIDS Behav. 2016;20(2):281-91. https://doi.or g/10.1007/s10461-015-1208-6.

12. da Cunha GH, Fiuza ML, Gir E, Aquino P, Pinheiro AK, Galvão MT. Quality of life of men with AIDS and the model of social determinants of health. Rev Lat Am Enfermagem. 2015;23(2):183-91. https://doi.org/10.159 0/0104-1169.0120.2541.

13. Liping M, Peng X, Haijiang L, Lahong J, Fan L. Quality of life of people living with HIV/AIDS: a cross-sectional study in Zhejiang Province, China. PLoS One. 2015;10(8):e0135705. https://doi.org/10.1371/journal.pone.0 135705.

14. Lan G-L, Yuan ZK, Clements-Nolle KD, Cook A, Yuan LL, Xu QY, et al. Social capital and quality of life among people living with HIV/AIDS in Southeast China. Asia Pacific J Public Health. 2016;28(4):325-35. https://d oi.org/10.1177/1010539516641490.

15. Zhakipbayeva BT, Nugmanova ZS, Tracy M, Birkhead GS, Akhmetova GM, DeHovitz J. Factors influencing the quality of life in persons living with human immunodeficiency virus infection in Almaty, Kazakhstan. Int J STD AIDS. 2019;30(13):1318-28. https://doi.org/10.1177/0956462419876484.

16. Dessie ZG, Zewotir T, Mwambi H, North D. Multivariate multilevel modeling of quality of life dynamics of HIV infected patients. Health Qual Life Outcomes. 2020;18(1):80. https://doi.org/10.1186/s12955-020-01330-2

17. Vo QT, Hoffman S, Nash D, El-Sadr WM, Tymejczyk OA, Gadisa T, et al. Gender differences and psychosocial factors associated with quality of life among ART initiators in Oromia, Ethiopia. AIDS Behav. 2016;20(8):1682-91. https://doi.org/10.1007/s10461-016-1396-8.

18. Mutabazi-Mwesigire DM, Martin F, Seeley J, Katamba A. Relationship between CD4 count and quality of life over time among HIV patients in Uganda: a cohort study. Health Qual Life Outcomes. 2015;13:144. https://doi.o $\mathrm{rg} / 10.1186 / \mathrm{s} 12955-015-0332-3$.

19. Bucciardini R, Pugliese K, Weimer L, Digregorio M, Fragola V, Mancini M, et al. Relationship between healthrelated quality of life measures and high HIV viral load in HIV-infected triple-class-experienced patients. HIV Clin Trials. 2014;15(4):176-83. https://doi.org/10.1310/hct1504-176.

20. Gutiérrez-Gabriel I, Godoy-Guinto J, Lucas-Alvarado H, Pineda-Germán B, Vázquez-Cruz E, Hernández-De la Rosa M, Sosa-Jurado F. Calidad de vida y variables psicológicas que afectan la adherencia al tratamiento antiretroviral en pacientes mexicanos con infección por VIH/SIDA. Rev Chilena Infectol. 2019;36(3):331-339. h ttps://doi.org/10.4067/S0716-10182019000300331.

21. Mutabazi-Mwesigire D, Katamba A, Martin F, Seeley J, Wu AW. Factors that affect quality of life among people living with HIV attending an urban clinic in Uganda: a cohort study. PLoS One. 2015;10(6). https://doi.org/ 10.1371/journal.pone.0126810.

22. Karkashadze E, Gates MA, Chkhartishvili N, DeHovitz J, Tsertsvadze T. Assessment of quality of life in people living with HIV in Georgia. Int J STD AIDS. 2017;28(7):672-8. https://doi.org/10.1177/0956462416662379. 
23. Pérez-Rodríguez I, Pérez-Salgado D, Compeán-Dardón MS, Staines-Orozco MG, Ortiz-Hernández L. Efectos secundarios del tratamiento antirretroviral y apego en pacientes con VIH de dos instituciones públicas. Med Int Méx. 2016;32(4):396-406. https://www.medigraphic.com/pdfs/medintmex/mim-2016/mim164d.pdf.

24. Zubaran C, Medeiros G, Foresti K, May W, Michelim L, Madi JM, et al. Quality of life and adherence to antiretroviral therapy in Southern Brazil. AIDS Care. 2014;26(5):619-25. https://doi.org/10.1080/09540121 .2013 .841838 .

25. Augustovski F, Lewin G, Elorrio E, Rubinstein A. The Argentine-Spanish SF-36 Health Survey was successfully validated for local outcome research. J Clin Epidemiol. 2008;61(12):1279-84. https://doi.org/10.1016/j.jcline pi.2008.05.004.

26. Canova-Barrios C, Oviedo-Santamaría P. Health-related quality of life among intensive care unit workers. Enferm Intensiva. 2021;32(4):181-188. https://doi.org/10.1016/j.enfi.2020.11.002.

27. Wickersham K, Sereika S, Kang H, Tamres L, Erlen J. Use of a Self-Report Medication Adherence Scale for Measuring Adherence to Antiretroviral Therapy in Patients With HIV/AIDS. J Nurs Meas. 2018;26(2):E72E88. https://doi.org/10.1891/1061-3749.26.2.E72.

28. Cardona-Duque D, Medina-Pérez O, Herrera-Castaño S, Orozco-Gómez P. Adherence to antiretroviral treatment and associated factors in people living with HIV/AIDS in Quindío, Colombia. Rev Fac Med. 2017;65(3):403-410. https://doi.org/10.15446/revfacmed.v65n3.55535

29. Instituto Nacional de estadísticas y Censos, República Argentina. Cuarta Encuesta Nacional de Factores de Riesgo (ENFR): Resultados definitivos [Internet]. 2018. https://www.indec.gob.ar/indec/web/Nivel4-Tema-4-32-68.

30. Guzmán VE, Echazu A, Vinciguerra M, Edelcopp A. Adherencia al tratamiento antirretroviral en pacientes trans [Internet]. 2019. https://infectologia.info/abstracts/adherencia-al-tratamiento-antirretroviral-en-pacientes-tra $\mathrm{ns} /$.

\section{Notas}

* Artículo original de investigación.

Licencia Creative Commons CC BY 4.0

Limitaciones: Las limitaciones del presente trabajo son la baja cantidad de observaciones (encuestados) y el hecho de que la población correspondía a características socioeconómicas de niveles medio en adelante, teniendo en cuenta que fueron seleccionados de una institución de carácter privado. Se sugiere para futuros estudios realizar trabajos comparativos entre poblaciones de distintos estratos socioeconómicos e incorporar un mayor número de sujetos para aumentar la potencia de los hallazgos.

Financiamiento: Ninguno.

Conflicto de intereses: Los autores declaran no tener conflicto de intereses.

Cómo citar este artículo: Canova Barrios C. J. Calidad de vida relacionada con la salud en sujetos con VIH. Investigación en Enfermería, Imagen y Desarrollo. 2021;23. https://doi.org/10.11144/Javeriana.ie23.cvrs 